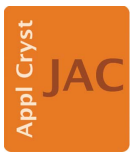

JOURNAL OF

APPLIED

CRYSTALLOGRAPHY

ISSN 1600-5767

Received 11 April 2018

Accepted 20 June 2018

Edited by K. Chapman, Argonne National

Laboratory, USA

Keywords: stacking disorder; polytypism; ice; total scattering.

Supporting information: this article has supporting information at journals.iucr.org/j

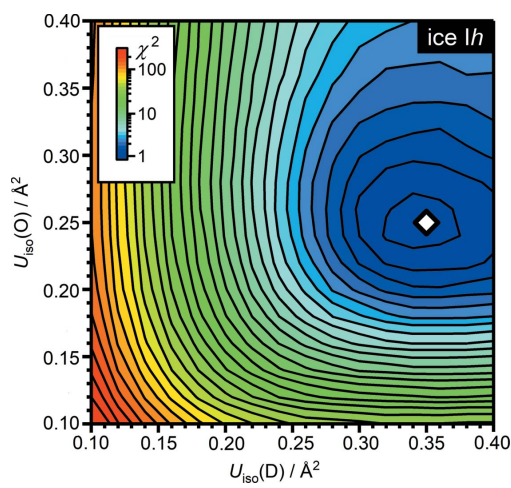

(C) 2018 International Union of Crystallography

\section{Analysis of stacking disorder in ice I using pair distribution functions}

\author{
Helen Y. Playford, ${ }^{a}$ Thomas F. Whale, ${ }^{b}$ Benjamin J. Murray, ${ }^{b}$ Matt G. Tucker ${ }^{c}$ and \\ Christoph G. Salzmann ${ }^{\mathrm{d} *}$
}

${ }^{\text {a } S T F C ~ I S I S ~ F a c i l i t y, ~ R u t h e r f o r d ~ A p p l e t o n ~ L a b o r a t o r y, ~ H a r w e l l ~ O x f o r d, ~ D i d c o t ~ O X 11 ~ 0 Q X, ~ U K, ~}{ }^{\mathbf{b}}$ Institute for Climate and
Atmospheric Science, School of Earth and Environment, University of Leeds, Leeds LS2 9JT, UK, ' Oak Ridge National
Laboratory, PO Box 2008, Oak Ridge, TN 37831, USA, and ${ }^{\mathbf{d}}$ Department of Chemistry, University College London,
London WC1H 0AJ, UK. *Correspondence e-mail: C.salzmann@ucl.ac.uk

Stacking-disordered materials display crystalline order in two dimensions but are disordered along the direction in which layered structural motifs are stacked. Countless examples of stacking disorder exist, ranging from close-packed metals, ice I and diamond to open-framework materials and small-molecule pharmaceuticals. In general, the presence of stacking disorder can have profound consequences for the physical and chemical properties of a material. Traditional analyses of powder diffraction data are often complicated by the presence of memory effects in the stacking sequences. Here it is shown that experimental pair distribution functions of stacking-disordered ice I can be used to determine local information on the fractions of cubic and hexagonal stacking. Ice is a particularly challenging material in this respect, since both the stacking disorder and the orientational disorder of the water molecules need to be described. Memory effects are found to contribute very little to the pair distribution functions, and consequently, the analysis of pair distribution functions is the method of choice for characterizing stacking-disordered samples with complicated and high-order memory effects. In the context of this work, the limitations of current structure-reconstruction approaches are also discussed.

\section{Introduction}

Stacking-disordered materials consist of layers of structural motifs that display periodicity within the plane of the layers. However, disorder arises because of the different ways the layers stack on top of one another, which can include rotations, translational displacements and mirror operations. Naturally, stacking disorder (SDO) is found for a wide range of layered materials, such as graphite (Li et al., 2009; Warner $e t$ al., 2009), $\mathrm{MoS}_{2}$ (Moser \& Lévy, 1994) and mica (Iijama \& Buseck, 1978), where there is stronger chemical bonding within the layers compared with the chemical interactions between the layers. An extreme case is turbostratic graphite where individual graphene sheets are stacked with significant rotational misalignment (Marchand, 1965). However, SDO can also be found for materials where the chemical bonding within the formal layer units is similar or even identical to that in the direction of stacking. The most fundamental and well known examples are close-packed metals and alloys, for which two geometric recipes of stacking exist based on face-centred cubic and hexagonal close packing (Edwards \& Lipson, 1942; Berliner \& Werner, 1986; Roy et al., 2014; Sławiński et al., 2018).

The 'ordinary' ice I consists of layers of hydrogen-bonded water molecules that form annulated six-membered rings in the armchair conformation. The thermodynamically stable 
form of ice I is hexagonal (ice $\mathrm{I} h$ ), which means that mirror images of the layers are placed on top of one another in an alternating fashion as shown in Fig. 1(a). However, cubic stacking of the layers is also possible, where identical layers are placed on top of one another but with displacements (see Fig. 1b). Hexagonal stacking leads to new six-membered rings between the layers in the boat conformation, whereas the armchair conformation is found in the case of cubic stacking. Consequently, a perfect cubic ice I structure (ice Ic) consists of only six-membered rings in the armchair conformation whereas ice $\mathrm{I} h$ contains a 50:50 mixture of boat and armchair rings.

Ice Ic was originally believed to form from low-temperature vapour deposition (König, 1943). However, more recently it has been shown that pure ice Ic has so far not been prepared and the metastable ice I samples contain varying degrees of cubic and hexagonal stacking, as shown in Fig. 1(c) (Kuhs et al., 1987, 2012; Hansen, Koza, Kuhs et al., 2008; Hansen, Koza Lindner et al., 2008; Malkin et al., 2012, 2015). Instead of calling this material ice Ic, the name stacking-disordered ice (ice Isd) has been suggested as a more accurate structural description (Malkin et al., 2012). To date, the most cubic bulk sample of ice Isd has been prepared by heating the ice II highpressure phase of ice at ambient pressure, which gave a percentage of cubic stacking events (or cubicity) in the ice Isd of $73.3 \%$ (Malkin et al., 2015). Freezing small droplets of liquid water in a vacuum has recently led to the formation of $\sim 20 \mathrm{~nm}$ ice Isd particles with $78 \%$ cubicity (Amaya et al., 2017).

Similar SDO phenomena have also been identified in diamond which is isostructural with ice I. However, here the fully cubic polytype is the most stable form and it has been shown that what were believed to be hexagonal diamond

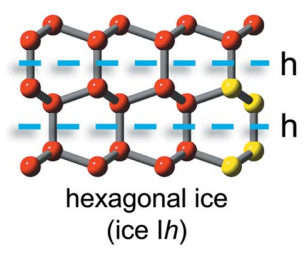

(a)

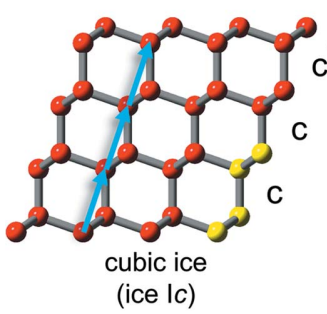

(b)

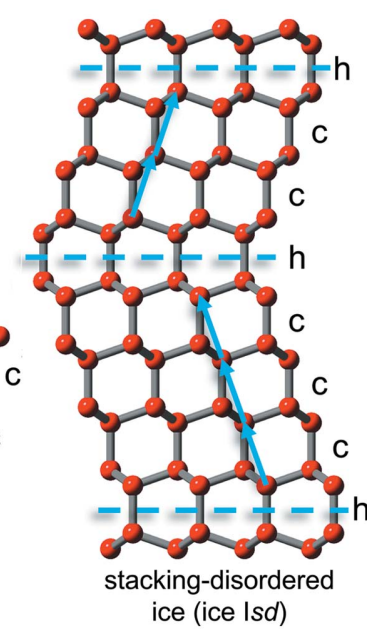

(c)
Figure 1

Stacking of layers in $(a)$ hexagonal, $(b)$ cubic and $(c)$ stacking-disordered ice I. Red spheres indicate oxygen atoms. Six-membered rings in the boat and armchair conformations are highlighted in yellow in $(a)$ and $(b)$, respectively. Hydrogen atoms have been omitted for clarity. Hexagonal or cubic stacking of layers is indicated by ' $h$ ' and 'c', respectively. Dashed lines show the mirror planes associated with hexagonal stacking whereas cubic stacking is highlighted by displacement vectors. samples (Bundy \& Kasper, 1967; Hanneman et al., 1967) should in fact be described as stacking-disordered diamond (Németh et al., 2014; Salzmann et al., 2015). The quest for fully hexagonal diamond is currently underway (Kraus et al., 2016, Shiell et al., 2016, Turneaure et al., 2017). Countless other examples of SDO exist across the materials world, including complex inorganic materials (Zimmermann \& Johnsson, 2015; Ainsworth et al., 2016) as well as zeolites and other openframework materials (Willhammar \& Zou, 2013). More recently, SDO has also been found in small-molecule pharmaceuticals such as aspirin, promethazine hydrochloride and aprepitant (Bond et al., 2007, Braun et al., 2008, 2017; Borodi et al., 2012, Price et al., 2016).

Depending on the material in question, the presence and extent of SDO can have profound consequences for its physical and chemical properties. In the case of ice, the extent of SDO has been found to affect its vapour pressure (Shilling et al., 2006), crystal shape (Murray, Salzmann et al., 2015), spectroscopic (Carr et al., 2014) and light scattering properties (Murray, Salzmann et al., 2015), and potentially surface chemistry (Behr et al., 2006). This may be important for understanding ice particles in and their impact on the Earth's atmosphere (Murray, Salzmann et al., 2015; Murray, Malkin \& Salzmann, 2015). For diamond, the hardness is thought to be influenced by the SDO, with fully hexagonal diamond potentially being much harder than its cubic counterpart (Pan et al., 2009; Qingkun et al., 2011). The bandgap and dielectric properties of diamond are also thought to depend on its cubicity (Gao, 2014). Furthermore, the solubility and hence the bioavailability of pharmaceuticals could be affected by the extent of SDO. In terms of material design, SDO therefore offers the fascinating prospect of fine-tuning the physical and chemical properties of materials by controlling the extent of SDO in a continuous fashion between extreme polytypic cases.

Given the widespread occurrence of SDO and the effect it can have on the properties of materials, it is highly desirable to have reliable and accurate methods available for the quantitative analysis of SDO. High-resolution transmission electron microscopy is widely used (Németh et al., 2014; Willhammar \& Zou, 2013). However, the individual observations in electron microscopy are not necessarily representative of the bulk sample and the analysis is dependent on favourable orientations of the investigated particles. The associated technique of selected area electron diffraction (SAED) is very powerful in the sense that it highlights the presence of SDO very clearly in the form of 'streaks' in diffraction images. Yet, again, the recorded images may not necessarily represent the bulk sample and the quantification of the extent of SDO can be difficult. Related to SAED are single-crystal X-ray and neutron diffraction, where streaking is also an indication of SDO.

Perfectly crystalline materials show diffraction intensity in the form of spots in reciprocal space, which are defined by their $h k l$ Laue indices where $h, k$ and $l$ are integers. The consequence of SDO is that some of the spots turn into streaks as one of the Laue indices is no longer required to be an integer and can take continuous values. For example, for close- 
packed metals $h k l$ reflections are affected by SDO when $(h-$ $k) / 3$ is not an integer (Lele et al., 1967; Prasad \& Lele, 1971).

The streaking in reciprocal space caused by SDO leads to asymmetric broadening, diffuse-scattering components and shifts of some of the Bragg peaks in X-ray and neutron powder diffraction. The diffraction patterns of stacking-disordered materials can be calculated, for example, using the DISCUS (Proffen \& Neder, 1997) and RMCProfile (Tucker et al., 2007) software packages. A recent update of the software TOPAS allows the refinement of structural parameters related to SDO (Coelho et al., 2016; Coelho, 2018). The DIFFaX approach is particularly elegant since it does not require the construction of a complete atomistic model representing a stacking-disordered material (Treacy et al., 1991). Instead, the input for DIFFaX contains information about the structures of the layers, the geometric recipes for stacking these layers and the associated stacking probabilities, which can take continuous values. Various $D I F F a X$-based software packages, such as DIFFaX + (Leoni et al., 2004), FAULTS (Casas-Cabanas et al., 2016) and MCDIFFaX (Salzmann et al., 2015; Malkin et al., 2015), that allow for the relevant structural parameters (including the stacking probabilities) to be refined have been presented.

In our MCDIFFaX analysis of ice I and diamond diffraction data, we implemented up to second-order memory effects for the stacking probabilities, which was necessary in some cases in order to obtain a good fit to the experimental diffraction data (Salzmann et al., 2015, Malkin et al., 2015). This means that independent stacking probabilities were defined which depend on the two previous stacking events. The four secondorder stacking probabilities for cubic stacking are $\Phi_{\text {ccc }}, \Phi_{\text {hcc }}$, $\Phi_{\text {chc }}$ and $\Phi_{\text {hhc }}$, where $\Phi_{\text {hcc }}$, for example, describes the probability of a cubic stacking event following (hc) stacking. Four related second-order stacking probabilities exist for hexagonal stacking: $\Phi_{\text {cch }}, \Phi_{\text {hch }}, \Phi_{\text {chh }}$ and $\Phi_{\text {hhh }}$. However, since the stacking of layers can only be either cubic or hexagonal, these can be calculated from the corresponding stacking probabilities for cubic stacking. For example, $\Phi_{\text {cch }}=1-\Phi_{\text {ccc. }}$. The first-order stacking probabilities, $\Phi_{\mathrm{cc}}$ and $\Phi_{\mathrm{hc}}$, can be obtained from the second-order probabilities using the following 'switching' equations:

$$
\begin{gathered}
\Phi_{\mathrm{cc}}=1-\Phi_{\mathrm{ch}}=1-\frac{\Phi_{\mathrm{cch}}}{\Phi_{\mathrm{cch}}+\Phi_{\mathrm{hcc}}}, \\
\Phi_{\mathrm{hc}}=\frac{\Phi_{\mathrm{hhc}}}{\Phi_{\mathrm{hhc}}+\Phi_{\mathrm{chh}}} .
\end{gathered}
$$

Finally, the zero-order cubicity, $\Phi_{c}$, which reflects the overall fraction of cubic stacking present in a sample, can be calculated from the first-order stacking probabilities:

$$
\Phi_{\mathrm{c}}=\frac{\Phi_{\mathrm{hc}}}{\Phi_{\mathrm{hc}}+\Phi_{\mathrm{ch}}} .
$$

The hexagonality, $\Phi_{\mathrm{h}}$, is then simply $1-\Phi_{\mathrm{c}}$.

To compare and discuss the extent of SDO in different samples, we have previously used so-called 'stackograms', as shown in Fig. 2 for ice Isd samples (Salzmann et al., 2015;
Malkin et al., 2015) In a stackogram, the first-order stacking probabilities are plotted against each other. The bottom lefthand corner, where both probabilities for cubic stacking are zero, corresponds to fully hexagonal ice $\mathrm{I} h$. Consequently, the fully cubic ice Ic state is located at the top right-hand corner. The diagonal line connecting the ice $\mathrm{I} h$ and ice $\mathrm{I} c$ corners is the line of random stacking since $\Phi_{\mathrm{cc}}=\Phi_{\mathrm{hc}}$. Along this line, the probability of cubic stacking does not depend on the previous stacking event. Moving upwards from this line, ice Isd with higher probabilities of clustering of the same type of stacking is found. The extreme case is the top left-hand corner, describing a physical mixture of pure ice $\mathrm{I} h$ and ice Ic. Below the random line, there is a higher tendency for switching between the two kinds of stacking. Ultimately, the most extreme case in the bottom right-hand corner is the perfectly alternating $(\mathrm{ch})_{x}$ polytype. Lines of constant cubicity emanate from the physical mixture corner and are shown as dashed lines in Fig. 2.

In the ideal case, the analysis of the diffraction data for a stacking-disordered material provides accurate information about the zero-order stacking probability (such as the cubicity) and also on the higher-order stacking probabilities if this is relevant for the sample in question. However, problems with fitting the diffraction data arise when high-order memory effects are present in the sample but not implemented in the analysis. This leads to incorrect values for all the determined

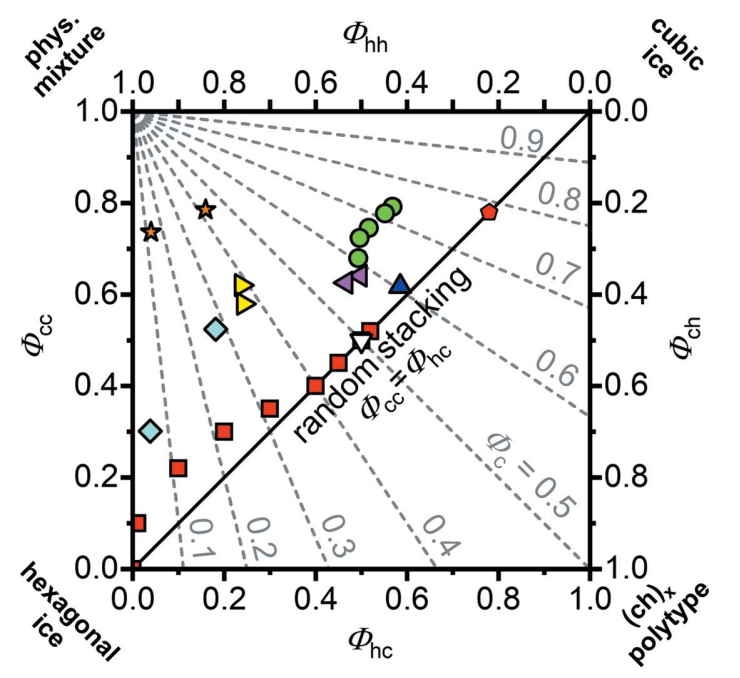

Figure 2

'Stackogram' used to describe the structure of stacking-disordered ice including first-order memory effects. The diagonal solid line indicates random stacking where $\Phi_{\mathrm{cc}}=\Phi_{\mathrm{hc}}$. Lines of constant cubicity $\left(\Phi_{\mathrm{c}}\right)$ are shown as dashed grey lines. All stacking probabilities shown in this plot were determined from the analysis of diffraction data. The ice I $s d$ samples were obtained by $\mathrm{D}_{2} \mathrm{O}$ vapour deposition at low temperatures (cyan diamonds) as well as ambient-pressure heating of $\mathrm{D}_{2} \mathrm{O}$ ice IX (yellow right-pointing triangles), $\mathrm{D}_{2} \mathrm{O}$ ice $\mathrm{V}$ (magenta left-pointing triangles), $\mathrm{D}_{2} \mathrm{O} \mathrm{CO}$ clathrate hydrate (orange stars) (Kuhs et al., 2012), $\mathrm{H}_{2} \mathrm{O}$ ice II (green circles) (Malkin et al., 2015) and $\mathrm{H}_{2} \mathrm{O}$ ice VIII (blue up-pointing triangle) (Shephard et al., 2016). $\mathrm{H}_{2} \mathrm{O}$ ice Isd obtained from homogenous and heterogenous freezing of water is indicated by the down-pointing open triangle and red squares, respectively (Malkin et al., 2012, 2015). The red pentagon corresponds to $\sim 20 \mathrm{~nm}$ ice Isd particles for which random stacking is assumed (Amaya et al., 2017). 
stacking probabilities. The inclusion of higher-order memory effects in the structural analysis is hampered by the fact that, for both ice I and diamond, the number of independent stacking probabilities increases exponentially with the order of the memory effects. In the worst cases, this could mean that the diffraction data cannot be usefully analysed at all. It is therefore highly desirable to have analytical tools available that are able to yield the zero-order stacking probability, such as the cubicity, in an accurate fashion even in the presence of complicated higher-order memory effects.

Here, we investigate whether stacking probabilities can be obtained from experimental pair distribution functions, and the approach is compared with the traditional analysis based on diffraction data. Since pair distribution functions represent real-space pair correlations and are particularly suitable for analysing short-range structural effects, it is anticipated that high-order memory effects should only contribute to the data at large distances and that it may therefore be possible to obtain accurate values for the zero-order stacking probability by analysing pair distribution functions at lower distances. Using this approach, the problems arising from high-order memory effects could be circumvented. Ice is a particularly challenging material for such analysis since both the SDO and the orientational disorder of the water molecules need to be described.

\section{Experimental and computational methods}

\subsection{Sample preparation and neutron diffraction experiments}

$\mathrm{D}_{2} \mathrm{O}$ ice VI samples were prepared by heating ice $\mathrm{I} h$ samples at $1.0 \mathrm{GPa}$ to $260 \mathrm{~K}$ in a piston-cylinder setup (Whale et al., 2013; Salzmann et al., 2016). The ice VI samples were recovered in liquid nitrogen and shipped to the ISIS Neutron and Muon Source. A fine powder of the ice samples was prepared using a porcelain pestle and mortar in liquid nitrogen and then transferred into a cylindrical $6 \mathrm{~mm}$ diameter vanadium can. The filled can was mounted onto a cryostat stick and quickly lowered into an AS Scientific 'Orange' cryostat precooled to $80 \mathrm{~K}$ at the POLARIS beamline. The identity of ice VI was confirmed by recording a diffraction pattern and the sample was subsequently heated until complete conversion to ice Isd was observed at $156 \mathrm{~K}$. After this, high-quality total scattering data of ice Is $d$ were collected at $80 \mathrm{~K}$. The ice Isd sample was annealed in the next step by heating to $201 \mathrm{~K}$, and total scattering data were then again recorded at $80 \mathrm{~K}$.

For comparison, ice $\mathrm{I} h$ was prepared by freezing droplets of $\mathrm{D}_{2} \mathrm{O}$ water in liquid nitrogen. A fine powder of ice $\mathrm{I} h$ was also transferred into a vanadium can and measured at $80 \mathrm{~K}$. Great care was taken to avoid overly compacting the ice $\mathrm{I} h$ inside the can to avoid preferred orientation effects. All three highquality data sets were collected for an integrated beam current of $1500 \mu \mathrm{A} \mathrm{h}$. Additionally, the empty beamline, an $8 \mathrm{~mm}$ diameter vanadium rod and an empty vanadium can were measured to normalize the data and subtract the contributions from the sample environment.
2.2. Data normalization and calculation of the total pair distribution function from the diffraction data

The POLARIS diffractometer collects scattered neutrons in the $13-160^{\circ}$ angle range, which covers a wavevector transfer range from $0.2 \AA$ to more than $50 \AA^{-1}$. The magnitude of the wavevector transfer $(Q)$ is calculated from the wavelength of the incident neutrons $(\lambda)$ and the associated scattering angles

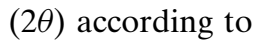

$$
Q=\frac{4 \pi}{\lambda} \sin \theta .
$$

The raw scattering data were normalized and corrected for absorption, multiple scattering and inelasticity effects, and the non-sample background scattering was subtracted using the GudrunN software package in order to obtain the total scattering structure factor $F(Q)$ (Soper, 2009). The experimental total pair distribution function $G(r)$ can be obtained from $F(Q)$ with a Fourier sine transform, where $\rho_{0}$ is the atomic number density and $r$ is the radial distance (Keen, 2001):

$$
G(r)=\frac{1}{(2 \pi)^{3} \rho_{0}} \int_{0}^{\infty} 4 \pi Q^{2} F(Q) \frac{\sin Q r}{Q r} \mathrm{~d} Q .
$$

$G(r)$ represents the sum of the individual partial radial distribution functions, $g_{i j}(r)$, for atom types $i$ and $j$ weighted by the mole fractions of the atoms, $c_{i}$, and their neutron scattering length, $b_{i}$ :

$$
G(r)=\sum_{i, j=0}^{n} c_{i} c_{j} b_{i} b_{j}\left[g_{i j}(r)-1\right] .
$$

The $g_{i j}(r)$ functions reflect the probabilities of finding an atom of type $j$ at a radial distance $r$ away from an atom of type $i$. In the case of $\mathrm{D}_{2} \mathrm{O}$ ice, the three partial radial distribution functions to consider are $g_{\mathrm{DD}}, g_{\mathrm{DO}}$ and $g_{\mathrm{OO}}$. Considering that $c_{\mathrm{D}}$ is greater than $c_{\mathrm{O}}$, and that the neutron scattering lengths of deuterium and oxygen are 6.671 and $5.803 \mathrm{fm}$, respectively, $g_{\text {DD }}$ is expected to make the strongest and $g_{\text {OO }}$ the weakest contribution to $G(r)$. The atomic number density for ice I at $80 \mathrm{~K}$ and ambient pressure was calculated from crystallographic data as $0.09365 \AA^{-3}$ (Röttger et al., 1994).

To highlight structural features in the 'intermediate' $r$ range, we used the experimental differential correlation function, $D(r)$, in the following analysis. $D(r)$ is related to $G(r)$ by

$$
D(r)=2 \pi r \rho_{0} G(r) \text {. }
$$

\subsection{Generation of ice Isd structural models}

A library of stacking-disordered ice I structures was generated computationally with the aim of identifying the one structure whose calculated $D(r)$ gives the best agreement with the experimental $D(r)$ data. Using the hexagonal unit cell of ice $\mathrm{I} h$, a $12 \times 12 \times 60$ supercell was generated with cell dimensions of $a=b=53.9652 \AA$ and $c=439.3320 \AA$. Creating a supercell in the $a$ and $b$ directions is necessary to be able to describe the hydrogen disorder present in the ice I samples. The hexagonal unit cell of ice $\mathrm{I} h$ contains two layers, and hence, there are 120 layers in the $12 \times 12 \times 60$ supercell. This 
large number of layers is required in order to realize a variety of different stacking regimes along the $c$ axis. An important side condition is that the various stacking sequences must be periodic across the boundaries of the supercell, as otherwise spurious features would be introduced into $D(r)$. To be able to realize fully hexagonal, fully cubic and strictly alternating (hc) $)_{x}$ stacking, the number of layers must be a multiple of 2,3 and 4, which is the case for structures with 120 layers.

The geometric reasons for these requirements become apparent when we consider how the layers must be stacked to build up an ice I structure. Fig. 3(a) shows two 'unit cells' of a single layer that are related to one another by mirror symmetry. To achieve hexagonal stacking, these cells are stacked on top of one another in an alternating fashion as shown in Fig. 3(b). In the case of cubic stacking, identical cells are stacked on top of one another, and horizontal shifts equal to a third of the $a$ and $b$ lattice parameters are performed in a diagonal fashion. It follows that fully hexagonal sequences must have an even number of layers, since a second mirror operation is necessary in order to return to the original structure. For a fully cubic structure, the number of layers needs to be a multiple of three to obtain periodic stacking since the shifts are always a third of the $a$ and $b$ lattice parameters. Note that the 'history' of hexagonal stacking determines the direction of the diagonal offsets of the layers upon subsequent cubic stacking, as shown in Fig. 3(b). This
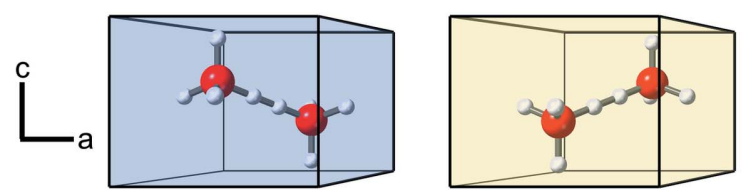

(a)
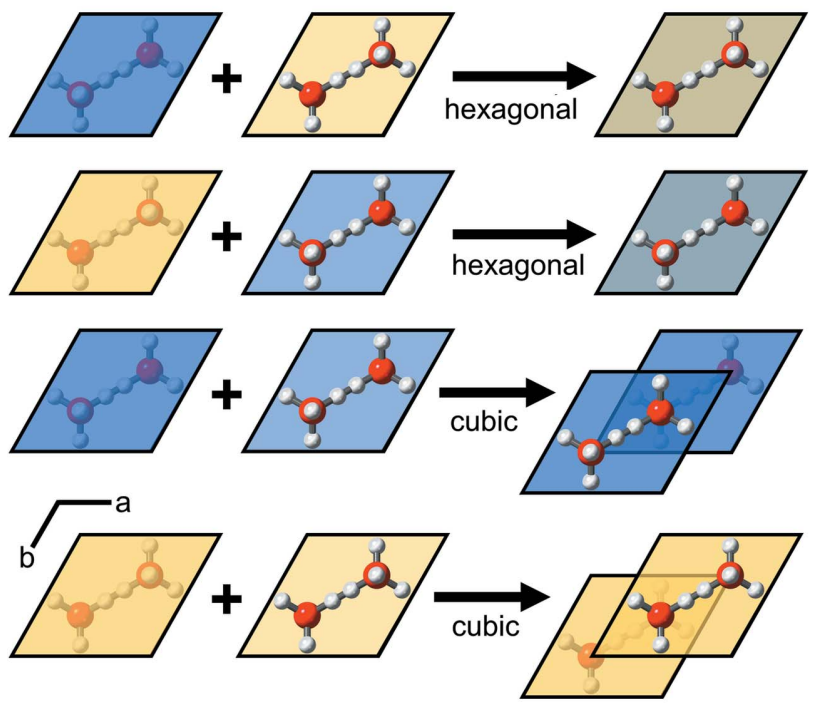

(b)

Figure 3

Geometric recipes for stacking the 'unit cells' of individual layers to achieve either cubic or hexagonal stacking with correct interlayer hydrogen bonding. (a) Individual building blocks that are related to one another by mirror symmetry viewed in the $a b$ plane. (b) Stacking of the building blocks shown in $(a)$ to give either hexagonal or cubic stacking. The direction of stacking is towards the reader. requirement can also be seen from the displacement vectors in Fig. 1(c). In this sense, it is now also clear that a strictly alternating $(\mathrm{hc})_{x}$ structure requires sequences of (hchc) blocks with four layers in order to be periodic.

Considering the geometric constraints for periodic stacking across the supercell, our program Stacky produces stackingdisordered structures with $n$ layers for target numbers of (cc) and (hc) stacking events, $n_{\mathrm{cc}}$ and $n_{\mathrm{hc}}$. Full details of this process and further discussions are given in Appendix $A$ of the supporting information. For 120 layers, Stacky identified 1752 periodic stacking sequences, which are indicated in Fig. 4(a). In Fig. 4(b), these structures are shown on a stackogram.

To obtain valid supercells with respect to the ice rules, hydrogen-ordered versions of the cells shown in Fig. 3 need to be used. This means that half of the hydrogen positions are defined as empty and half as occupied such that only $\mathrm{H}_{2} \mathrm{O}$ molecules are found and all hydrogen bonds contain one occupied and one empty position. In the final step, all 1762 stacking-disordered structures generated by Stacky were hydrogen disordered using our program RandomIce (Salzmann et al., 2016).
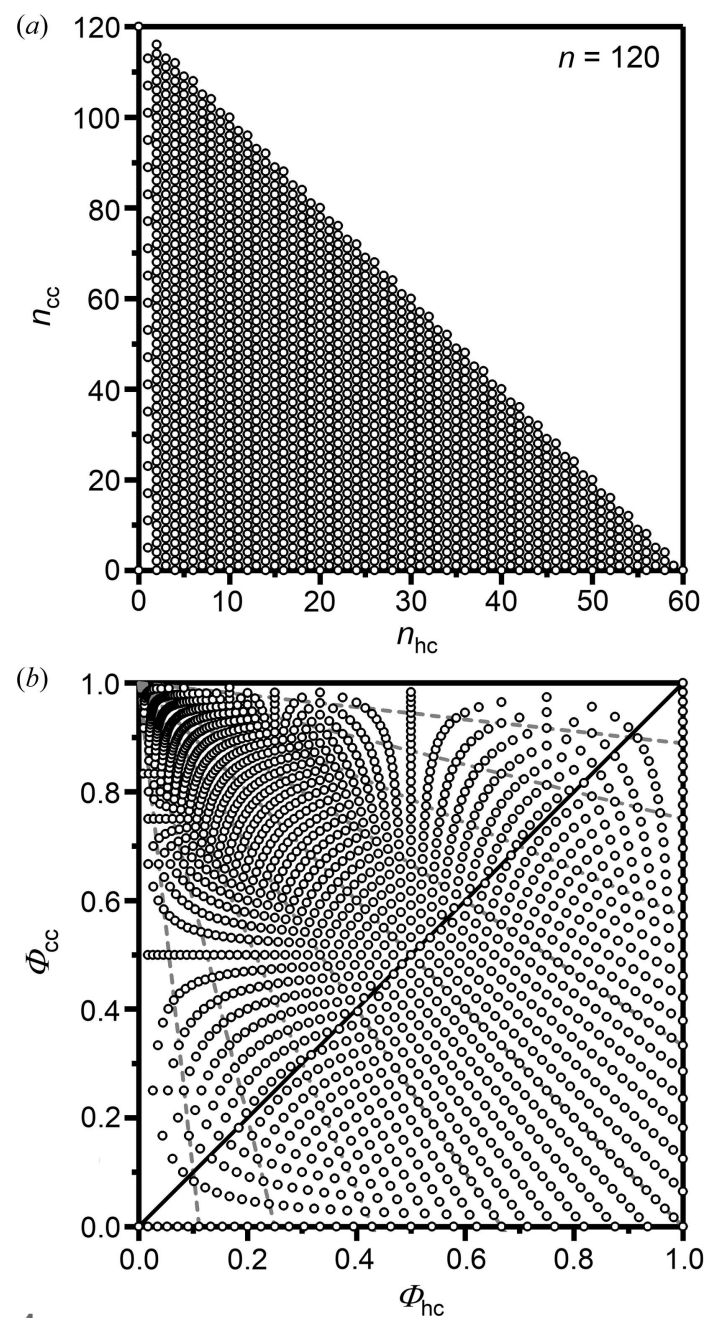

Figure 4

(a) Possible values of the numbers of (cc) and (hc) stacking events, $n_{\mathrm{cc}}$ and $n_{\text {hc }}$, in a stacking sequence with 120 layers. (b) Corresponding stacking probabilities indicated on the stackogram. 


\subsection{Calculation of pair distribution functions}

The $D(r)$ total distribution functions of the library structures were calculated using the RMCProfile software suite (Tucker et al., 2007). Atomic displacements of the O and D atoms were achieved with the gaussdist subroutine, which randomly displaces atoms with Gaussian probabilities in line with a target isotropic atomic displacement parameter $U_{\text {iso. }}$. The hexagonal symmetry of the $12 \times 12 \times 60$ supercell gives an $r_{\text {max }}$ of $a \sin \left(60^{\circ}\right) / 2=23.3676 \AA$ with $a=53.9652 \AA$. For distances beyond $r_{\max }$, the same atoms would be counted more than once during the calculation of $D(r)$, which is therefore only defined in the 0 to $r_{\max }$ distance range. Fig. S1 in Appendix $B$ of the supporting information shows the $D(r)$ functions for three independently generated hydrogen-disordered structures with $\Phi_{\mathrm{cc}}=\Phi_{\mathrm{hc}}=0.5$. This shows that different structures located at the same point on the stackogram give very similar $D(r)$ data for our chosen size of the supercell.

\section{Results and discussion}

The normalized $F(Q)$ diffraction data of ice $\mathrm{I} h$ as well as of the two ice Isd samples are shown in Fig. 5(a). The ice Isd sample obtained immediately after the phase transition from ice VI $\left(T_{\mathrm{A}}=156 \mathrm{~K}\right)$ shows an absence of some of the Bragg peaks expected for ice $\mathrm{I} h$ as well as the characteristic diffuse scattering around the cubic 111 peak around $1.7 \AA^{-1}$. This is consistent with ice Isd containing significant amounts of cubic stacking. Annealing the ice Isd sample at $201 \mathrm{~K}$ leads to increased intensities of Bragg peaks related to ice $\mathrm{I} h$. But, the intensities do not yet reach the values expected for pure ice $\mathrm{I} h$, indicating that cubic stacking is still present in the sample.

The corresponding experimental $D(r)$ total distribution functions are shown in Fig. 5(b). The low- $r$ regions of the three samples are very similar as expected, because of the identical nearest-neighbour environments in the ice I family. The various low- $r$ peaks are assigned starting from the $\mathrm{O}-\mathrm{D}$ distance at $1.0 \AA$ to the hydrogen-bonded $\mathrm{O}$. . O distance at $2.75 \AA$. Differences arising from cubic and hexagonal stacking are expected to manifest at distances above the average layer separation of $3.7 \AA$. Yet, the differences in $D(r)$ are quite small initially up to the negative dip at $6.6 \AA$. Above this, pronounced differences between ice $\mathrm{I} h$ and the two ice Isd samples are found, the most notable being the peak at $8.5 \AA$ which is most intense for ice $\mathrm{I} h$. Another peak at around $12.1 \AA$ also seems to be quite responsive to the amount of cubic/hexagonal stacking and is most intense for the ice Isd sample expected to contain the most cubic stacking.

The $D(r)$ functions calculated from the various library structures would yield very sharp peaks since the structures have been built up from the average crystal structure. To broaden the features in the calculated $D(r)$ functions, the $\mathrm{O}$ and $\mathrm{D}$ atoms were randomly and isotropically displaced in line with target atomic displacement parameters, $U_{\text {iso }}(\mathrm{O})$ and $U_{\text {iso }}(\mathrm{D})$, respectively. This approach can only be used across a relatively limited $r$ range since it does not take into account the correlations in the displacements that exist between neighbouring atoms. Since the 7-16 $\AA$ range in $D(r)$ seems to contain several features responsive to the amount of cubic/ hexagonal stacking, the $U_{\text {iso }}$ parameters were systematically mapped from 0.1 to $0.4 \AA^{2}$ using the $(\mathrm{h})_{120}$ library structure and experimental $D(r)$ data of ice $\mathrm{I} h$.

Fig. 6 shows a contour plot of the goodness of fit $\left(\chi^{2}\right)$ against the $U_{\text {iso }}$ parameters of the $\mathrm{O}$ and $\mathrm{D}$ atoms. The best fit to the experimental $D(r)$ data is found for $U_{\text {iso }}(\mathrm{O})=0.25 \AA^{2}$ and $U_{\text {iso }}(\mathrm{D})=0.35 \AA^{2}$, and these values will be used in the following for the ice Isd library structures. A larger atomic displacement parameter for $\mathrm{D}$ compared with $\mathrm{O}$ is expected owing to the smaller mass of $\mathrm{D}$, but it is probably also a consequence of the greater positional disorder of the deuterium atoms in the hydrogen-disordered ice.

The fit of the experimental ice $\mathrm{I} h D(r)$ data using the ice $\mathrm{I} h$ structural model and the optimal $U_{\text {iso }}$ parameters is shown in Fig. 7(a).
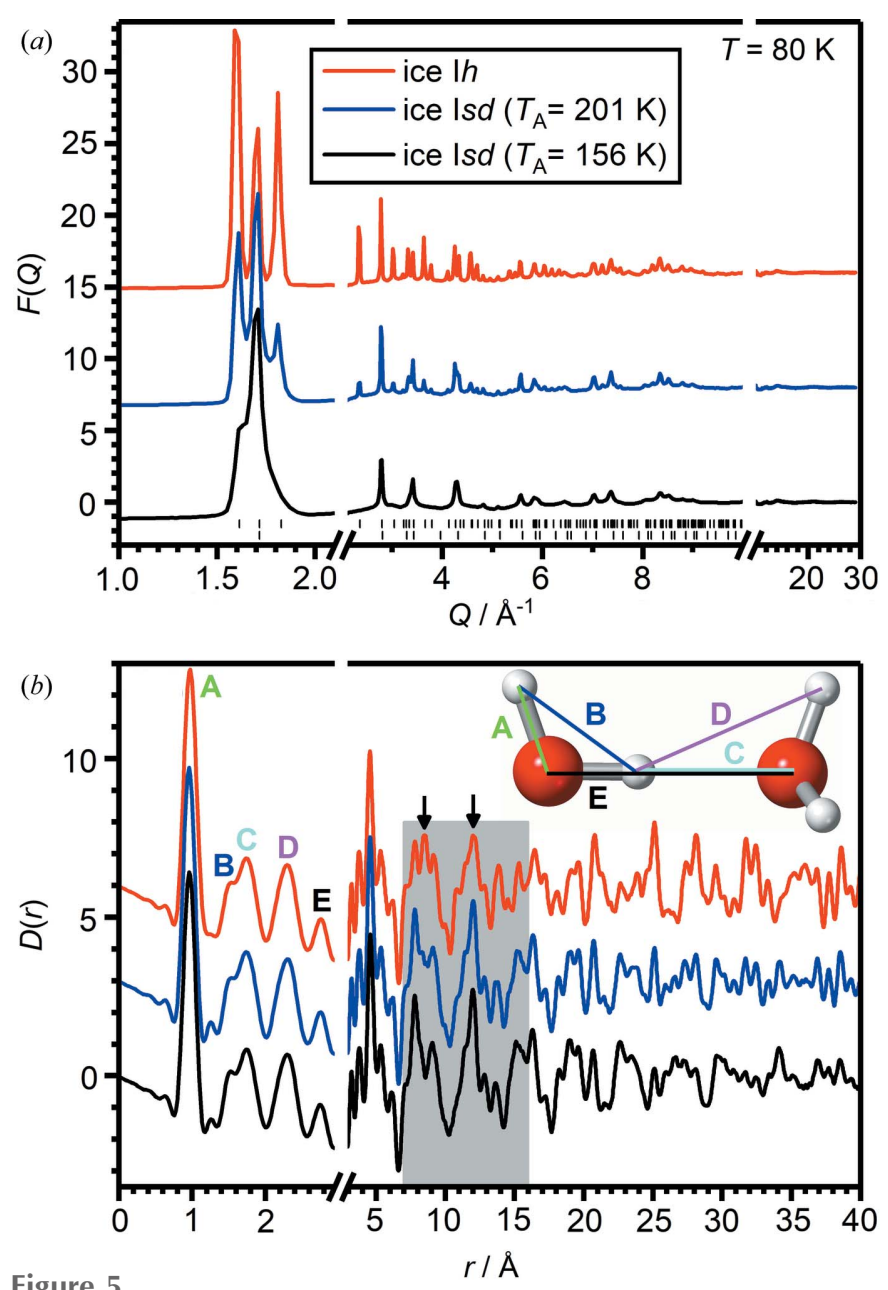

Figure 5

(a) Experimental $F(Q)$ diffraction data collected at $80 \mathrm{~K}$ for ice $\mathrm{I} h$ as well as two ice Isd samples annealed at 156 and $201 \mathrm{~K}$, respectively. Tickmarks indicate the expected positions of Bragg peaks for ice $\mathrm{I} h$ (top) and ice Ic (bottom) for $Q<10 \AA^{-1}$. (b) Corresponding $D(r)$ data, with the features in the low- $r$ region assigned to the intra- and intermolecular distances as indicated. The $r$ range used for fitting is indicated by a grey shaded area and the arrows indicate the major differences in this range. The red and blue curves in $(a)$ and $(b)$ are shifted vertically for clarity. 
The impact of hydrogen order/disorder, cubicity and firstorder memory effects in the stacking sequence on the calculated $D(r)$ data is investigated next. Fig. 8(a) shows the calculated $D(r)$ functions for hydrogen-ordered and hydrogen-disordered ice $\mathrm{I} h$. The nearest-neighbour environments are expected to be very similar, and consequently, differences between the two functions are only observed above $\sim 4 \AA$. However, though the differences are overall relatively small, the influence on the peak intensity, for example at $\sim 12 \AA$, illustrates the importance of implementing hydrogen disorder in the library structures. Incidentally, the calculated $D(r)$ function of the hydrogen-disordered ice $\mathrm{I} h$ illustrates that the hydrogen disordering with our RandomIce program was successful. Mistakes with the hydrogen disordering would manifest in a peak at $0.75 \AA$ corresponding to two occupied hydrogen sites along the same hydrogen bond.

Fig. $8(b)$ shows the calculated $D(r)$ functions for ice $\mathrm{I} h$, randomly stacked ice Is $d$ with a cubicity of 0.5 and ice Ic. So, this comparison highlights the changes in $D(r)$ along the random-stacking line in the stackogram and therefore the influence of the cubicity $\Phi_{c}$. In line with the experimental data shown in Fig. 5(b), pronounced differences between the three functions start to appear above the negative dip at $6.6 \AA$ and there do not seem to be any systematic increases in the differences above this value.

The effects of differences in the first-order stacking probabilities on $D(r)$ at a constant cubicity of 0.5 are shown in Fig. $8(\mathrm{c})$. The corresponding structures are the ones found along the 0.5 cubicity line, including the $(\mathrm{h})_{60}(\mathrm{c})_{60}$ structure containing two slabs of hexagonal and cubic stacking, a randomly stacked structure, and the strictly alternating $(\mathrm{hc})_{60}$ structure. Overall, the differences between the three $D(r)$ functions are much smaller than those shown in Fig. 8(b). Small differences only emerge above $9 \AA$. It is emphasized that these differences are as pronounced as they can be as a

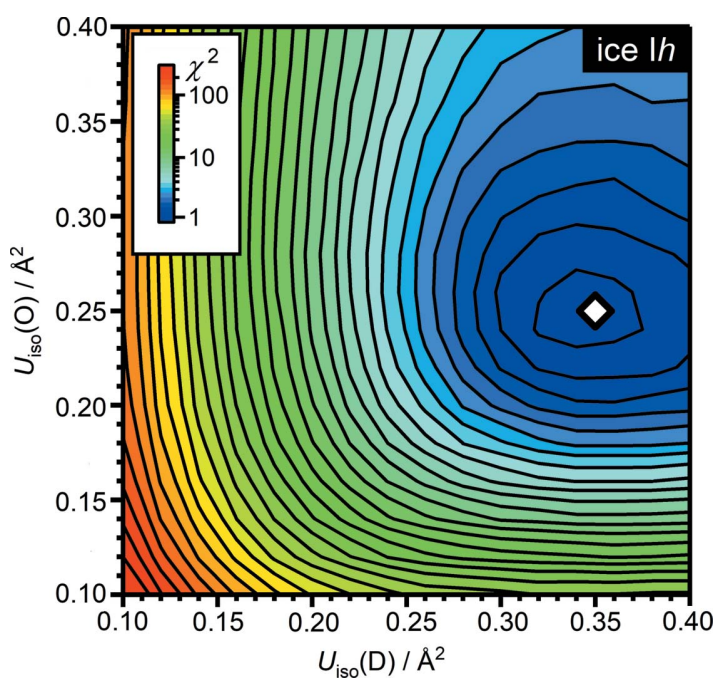

Figure 6

Goodness of fit $\left(\chi^{2}\right)$ of the ice $\mathrm{I} h D(r)$ data fitted with an ice $\mathrm{I} h$ structural model in the 7-16 A range by varying the isotropic atomic displacement of the $\mathrm{O}$ and $\mathrm{D}$ atoms. The open diamond indicates the location of the best fit at $U_{\text {iso }}(\mathrm{O})=0.25 \AA$ and $U_{\text {iso }}(\mathrm{D})=0.35 \AA^{2}$.
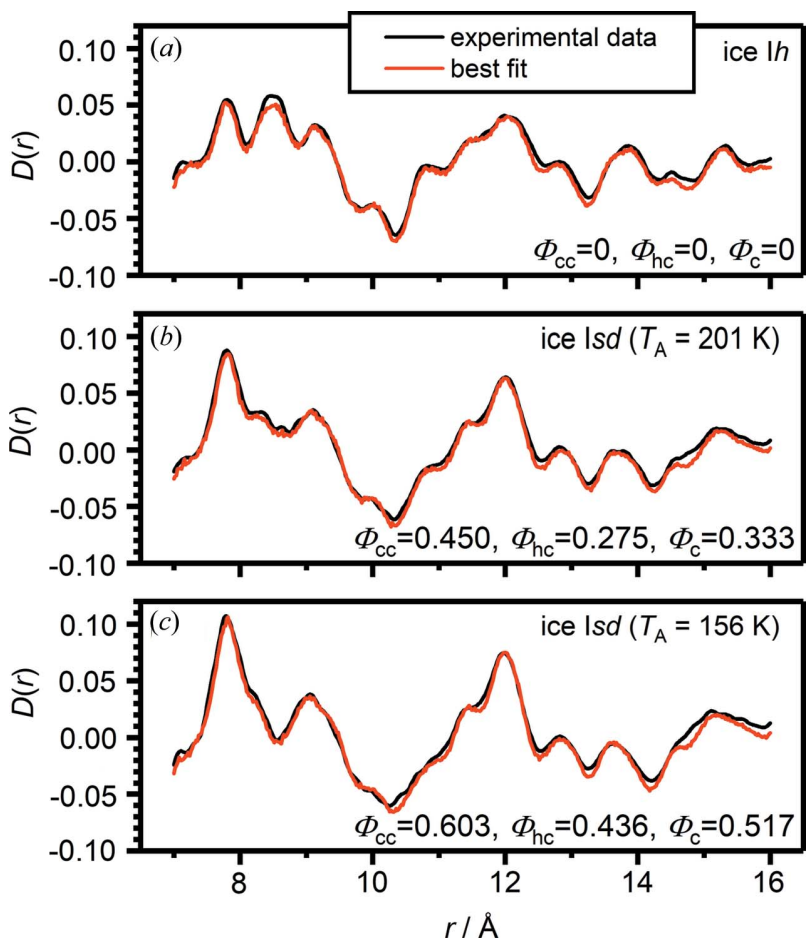

Figure 7

Best fits of the $D(r)$ data in the 7-16 $⿱$ range of $(a)$ ice $\mathrm{I} h,(b)$ ice Isd annealed at $201 \mathrm{~K}$ and $(c)$ ice Isd annealed at $158 \mathrm{~K}$. The stacking probabilities of the best-fitting library structures are given in each panel. The optimal atomic displacement parameters as determined for ice $\mathrm{I} h$ were also used for fitting the $D(r)$ data of the two ice Isd samples $\left[U_{\text {iso }}(\mathrm{O})=0.25 \AA^{2}\right.$ and $\left.U_{\text {iso }}(\mathrm{D})=0.35 \AA^{2}\right]$.
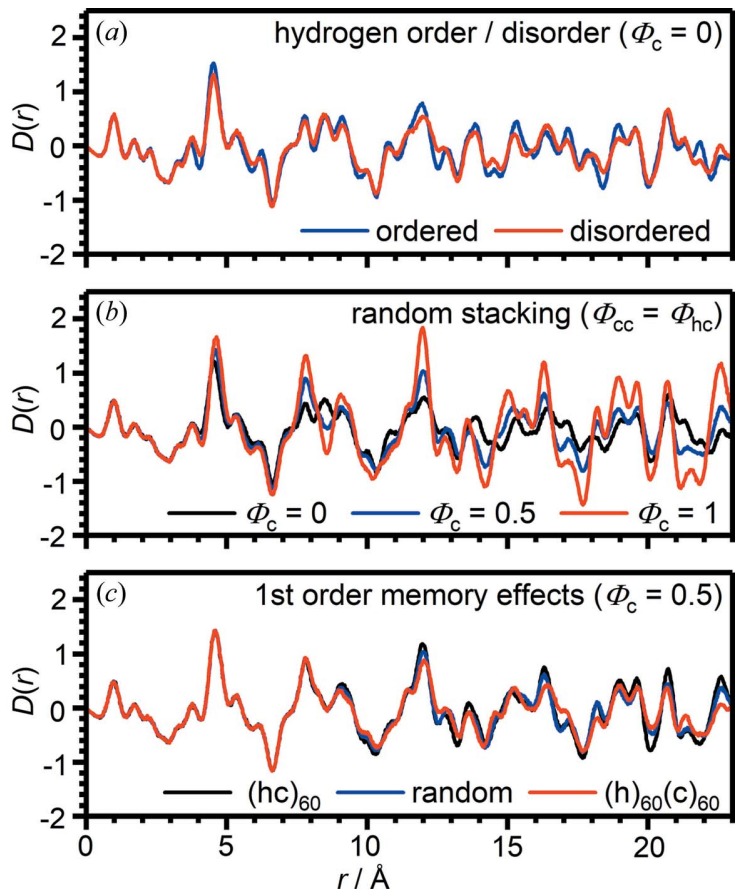

Figure 8

Influences of structural characteristics on the calculated $D(r)$ data. Comparisons of $(a)$ hydrogen-ordered/disordered ice $\mathrm{I} h,(b)$ hydrogendisordered ice I with cubicities of $0,0.5$ (random stacking) and 1, and $(c)$ hydrogen-disordered ice I with cubicities of 0.5 but differences in the firstorder memory effects (strictly alternating, random stacking, and two 'slabs' of cubic and hexagonal stacking). 
consequence of different first-order memory effects. This is because the most pronounced changes in both first-order stacking probabilities, $\Phi_{\mathrm{cc}}$ and $\Phi_{\mathrm{hc}}$, are observed along the 0.5 cubicity line. In conclusion, these comparisons show that the zero-order stacking probability, i.e. the cubicity, is much more robustly encoded in $D(r)$ than the first-order stacking probabilities. This of course makes sense since the cubicity is a more local structural descriptor than the first-order stacking probabilities.

The best fits to the experimental $D(r)$ data of the two ice Isd samples were found by calculating the goodness of fit for all library structures. The resulting $\chi^{2}$ values are shown in the stackograms in Fig. 9, which also include the $\chi^{2}$ values with respect to fitting the ice $\mathrm{I} h D(r)$ data.

There are clear minima in all three cases, in particular when considering that the $\chi^{2}$ values are shown on a logarithmic scale in Fig. 9. For the ice $\mathrm{I} h$ sample, the minimum is found at $\Phi_{\mathrm{cc}}=$ 0 and $\Phi_{\mathrm{hc}}=0.0167$, which corresponds to the library structure immediately next to the fully hexagonal $(\mathrm{h})_{120}$ structure with a cubicity of 0.0167 . Considering that the ice $\mathrm{I} h$ was made by rapidly freezing water in liquid nitrogen, the presence of a small percentage of cubic stacking is possible. However, owing to the relatively small number of library structures for $\Phi_{\mathrm{c}}<$ 0.1 , the actual cubicity may well lie below 0.0167 . Furthermore, the small cubicity value could potentially be an artefact arising from a small degree of preferred orientation in the ice I $h$ sample. The best fit for the ice Is $d$ sample annealed at $156 \mathrm{~K}$ is found at $\Phi_{\mathrm{cc}}=0.4355, \Phi_{\mathrm{hc}}=0.6034$ and $\Phi_{\mathrm{c}}=0.5167$. The ice Isd sample annealed at $201 \mathrm{~K}$ shows, as expected, a decrease in the cubicity to $\Phi_{\mathrm{c}}=0.3333$, calculated from $\Phi_{\mathrm{cc}}=0.45$ and $\Phi_{\mathrm{hc}}=0.275$.

In addition to the comparisons shown in Fig. 8 , the $\chi^{2}$ mapping in Fig. 9 illustrates that the zero-order cubicity information is more strongly encoded in $D(r)$ than the firstorder memory effects. The best-fit minima are very localized with respect to cubicity, as illustrated by the fact that the contour lines run mostly parallel to the lines of constant cubicity ( $c f$. Fig. 2). Along the lines of constant cubicity, as indicated by the white-dashed lines in Fig. 9, the minima with respect to the best fit are much more shallow. This reflects the fact that the information on the first-order stacking probabilities contributes much less to $D(r)$ than the cubicity, at least in the $r$ range used by us. In fact, it is questionable if the sample after annealing at $156 \mathrm{~K}$ has first-order stacking probabilities that are located below the random-stacking line in the stackogram. So far, such a state of SDO, which would indicate a preferred tendency for switching between cubic and hexagonal stacking, has not been identified for ice or diamond (Malkin et al., 2015, Salzmann et al., 2015).

In summary, we have demonstrated that the cubicity of stacking-disordered ice I samples can be determined from experimental total distribution functions. Ice is a particularly challenging material in this respect since both the SDO and the orientational disorder of the water molecules have to be realized in the library structures. The more long-range structural information associated with first-order stacking probabilities contributes significantly less to $D(r)$ than the cubicity, and therefore it seems difficult to obtain accurate values for $\Phi_{\mathrm{cc}}$ and $\Phi_{\mathrm{hc}}$. In this context, it is interesting to compare the $\chi^{2}$ mapping shown in Fig. 9 with that in Fig. 10 of Malkin et al. (2015), which was prepared by calculating diffraction patterns
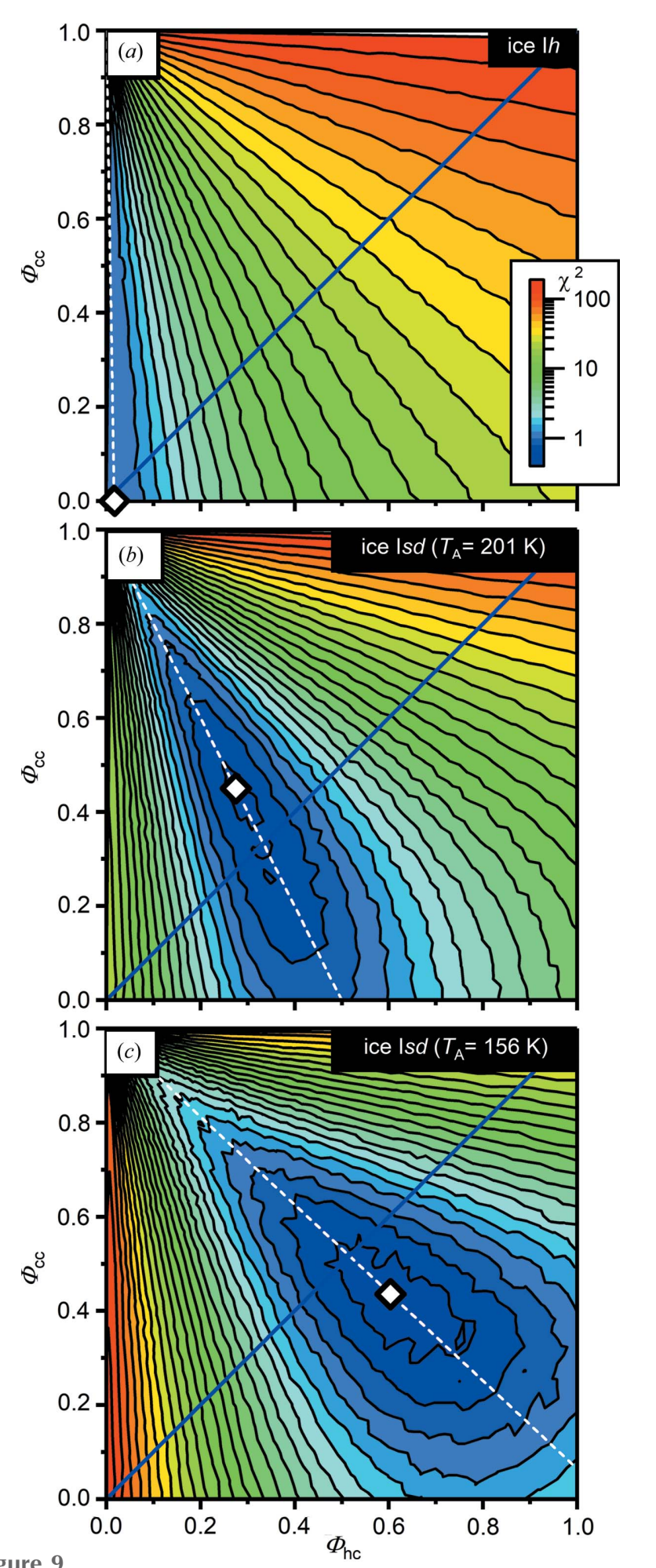

Figure 9

Goodness of fit $\left(\chi^{2}\right)$ of the library structures with respect to the three experimental $D(r)$ data in the 7-16 $\AA$ range. The open diamonds indicate the positions of the best fits. Dashed white lines indicate the cubicities of the best fits, which are (a) 0.0167, (b) 0.3333 and (c) 0.5167 . 
with DIFFaX. The best fit determined using diffraction data is less well located with respect to cubicity compared with the $D(r)$ analysis presented here. The sensitivity for cubicity compared with the first-order stacking probabilities is only slightly larger in the case of the diffraction analysis. This means that the $\chi^{2}$ 'basin' in the stackogram is more uniform in all directions in the case of diffraction data, and it is therefore easier to obtain more accurate values for the first-order stacking probabilities. On the other hand, the $D(r)$ analysis holds in principle the prospect of obtaining highly accurate cubicity values. In the presence of complex high-order memory effects, the $D(r)$ analysis would certainly be the method of choice. Furthermore, an additional advantage of the $D(r)$ analysis is that it circumvents the need for complicated profile functions such as the ones required for time-offlight neutron diffraction.

A final aspect of our study is that it highlights the shortcomings of current structure-reconstruction software packages like RMCProfile, which operate by moving individual atoms or molecules in real space. Starting from hydrogen-ordered ice $\mathrm{I} h$, it would be very difficult if not impossible to arrive at a structure that successfully describes the SDO as well as hydrogen disorder of an ice Is $d$ sample. This would require collective movements of entire layers as well as highly cooperative molecular reorientations that obey the ice rules. The movement of layers and the orientational disorder of the water molecules are of course highly correlated, since a unique 'fingerprint' pattern exists between the layers in terms of the directionalities of the hydrogen bonds. This would be far beyond the capabilities of atomistic Monte Carlo based 'random moves' and this is the reason why we have chosen to use a large library of structures to find the best fit to the $D(r)$ data of ice Isd, rather than attempting a full reverse Monte Carlo refinement. However, future efforts should be directed towards developing structure-reconstruction approaches that are not based on moving individual atoms but treat entire cooperative structural rearrangements as degrees of freedom. First steps towards this goal were the use of local distortion modes for the analysis of pair distribution data of $\mathrm{BaTiO}_{3}$ (Senn et al., 2016) and the implementation of rigid bodies and symmetry modes in the TOPAS software to reduce the degrees of freedom in the structural models (Coelho et al., 2015). The description of stacking disorder with the help of Markov chains has very recently been reviewed (Hart et al., 2018).

\section{Acknowledgements}

We thank the ISIS Neutron and Muon source for access to the POLARIS beamline and Sarah L. Price for helpful discussions. We are grateful to T. Malkin and A. Barlow for help during the ISIS experiment.

\section{Funding information}

We thank the Royal Society for a University Research Fellowship (CGS, UF150665), the Natural Environment Research Council (studentship No. NE/I019057/1 to TFW) and the European Research Council (grant No. 240449 ICE to BM; grant No. 648661 MarineIce to BM). This project has also received funding from the European Research Council under the European Union's Horizon 2020 research and innovation programme (grant No. 725271 to CGS).

\section{References}

Ainsworth, C. M., Lewis, J. W., Wang, C.-H., Coelho, A. A., Johnston, H. E., Brand, H. E. A. \& Evans, J. S. O. (2016). Chem. Mater. 28, 3184-3195.

Amaya, A. J. et al. (2017). J. Phys. Chem. Lett. 8, 3216-3222.

Behr, P., Terziyski, A. \& Zellner, R. (2006). J. Phys. Chem. A, 110, 8093-8107.

Berliner, R. \& Werner, S. A. (1986). Phys. Rev. B, 34, 3586-3603.

Bond, A. D., Boese, R. \& Desiraju, G. R. (2007). Angew. Chem. Int. Ed. 46, 618-622.

Borodi, G., Pop, M. M., Onija, O. \& Filip, X. (2012). Cryst. Growth Des. 12, 5846-5851.

Braun, D. E., Gelbrich, T., Kahlenberg, V., Laus, G., Wieser, J. \& Griesser, U. J. (2008). New J. Chem. 32, 1677-1685.

Braun, D. E., Lingireddy, S. R., Beidelschies, M. D., Guo, R., Müller, P., Price, S. L. \& Reutzel-Edens, S. M. (2017). Cryst. Growth Des. 17, 5349-5365.

Bundy, F. P. \& Kasper, J. S. (1967). J. Chem. Phys. 46, 3437-3446.

Carr, T. H. G., Shephard, J. J. \& Salzmann, C. G. (2014). J. Phys. Chem. Lett. 5, 2469-2473.

Casas-Cabanas, M., Reynaud, M., Rikarte, J., Horbach, P. \& Rodríguez-Carvajal, J. (2016). J. Appl. Cryst. 49, 2259-2269.

Coelho, A. A. (2018). J. Appl. Cryst. 51, 210-218.

Coelho, A. A., Chater, P. A. \& Kern, A. (2015). J. Appl. Cryst. 48, 869-875.

Coelho, A. A., Evans, J. S. O. \& Lewis, J. W. (2016). J. Appl. Cryst. 49, 1740-1749.

Edwards, O. S. \& Lipson, H. (1942). Proc. R. Soc. London Ser. A, 180, 268-277.

Gao, S.-P. (2014). Phys. Status Solidi B, 252, 235-242.

Hanneman, R. E., Strong, H. M. \& Bundy, F. P. (1967). Science, 155, 995-997.

Hansen, T. C., Koza, M. M. \& Kuhs, W. F. (2008). J. Phys. Condens. Matter, 20, 285104.

Hansen, T. C., Koza, M. M., Lindner, P. \& Kuhs, W. F. (2008). J. Phys. Condens. Matter, 20, 285105.

Hart, A. G., Hansen, T. C. \& Kuhs, W. F. (2018). Acta Cryst. A74, 357372.

Iijama, S. \& Buseck, P. R. (1978). Acta Cryst. A34, 709-719.

Keen, D. A. (2001). J. Appl. Cryst. 34, 172-177.

König, H. (1943). Z. Kristallogr. 105, 279-286.

Kraus, D. et al. (2016). Nat. Commun. 7, 10970.

Kuhs, W. F., Bliss, D. V. \& Finney, J. L. (1987). J. Phys. Colloq. C1, 48, 631-636.

Kuhs, W. F., Sippel, C., Falenty, A. \& Hansen, T. C. (2012). Proc. Natl Acad. Sci. USA, 109, 21259-21264.

Lele, S., Anantharaman, T. R. \& Johnson, C. A. (1967). Phys. Status Solidi (b), 20, 59-68.

Leoni, M., Gualtieri, A. F. \& Roveri, N. (2004). J. Appl. Cryst. 37, 166-173.

Li, H., Yang, C. \& Liu, F. (2009). Sci. China Chem. 52, 174-180.

Malkin, T. L., Murray, B. J., Brukhno, A. V. J. A., Anwar, J. \& Salzmann, C. G. (2012). Proc. Natl Acad. Sci. USA, 109, 1041-1045.

Malkin, T. L., Murray, B. J., Salzmann, C. G., Molinero, V., Pickering, S. J. \& Whale, T. F. (2015). Phys. Chem. Chem. Phys. 17, 60-76.

Marchand, A. (1965). Les Carbones, edited by A. Pacault, pp. 232247. Paris: Masson.

Moser, J. \& Lévy, F. (1994). Thin Solid Films, 240, 56-59.

Murray, B. J., Malkin, T. L. \& Salzmann, C. G. (2015). J. Atmos. Sol.Terrestrial Phys. 127, 78-82. 
Murray, B. J., Salzmann, C. G., Heymsfield, A. J., Dobbie, S., Neely, R. R. \& Cox, C. J. (2015). Bull. Am. Meteor. Soc. 96, 1519-1531.

Németh, P., Garvie, L. A. J., Aoki, T., Dubrovinskaia, N., Dubrovinsky, L. \& Buseck, P. R. (2014). Nat. Commun. 5, 5447.

Pan, Z., Sun, H., Zhang, Y. \& Chen, C. (2009). Phys. Rev. Lett. 102, 055503.

Prasad, B. \& Lele, S. (1971). Acta Cryst. A27, 54-64.

Price, S. L., Braun, D. E. \& Reutzel-Edens, S. M. (2016). Chem. Commun. 52, 7065-7077.

Proffen, T. \& Neder, R. B. (1997). J. Appl. Cryst. 30, 171-175.

Qingkun, L., Yi, S., Zhiyuan, L. \& Yu, Z. (2011). Scr. Mater. 65, 229232.

Röttger, K., Endriss, A., Ihringer, J., Doyle, S. \& Kuhs, W. F. (1994). Acta Cryst. B50, 644-648.

Roy, B., Kumar, N. K., Nambissan, P. M. G. \& Das, J. (2014). AIP Adv. 4, 067101.

Salzmann, C. G., Murray, B. J. \& Shephard, J. J. (2015). Diamond Relat. Mater. 59, 69-72.

Salzmann, C. G., Slater, B., Radaelli, P. G., Finney, J. L., Shephard, J. J., Rosillo-Lopez, M. \& Hindley, J. (2016). J. Chem. Phys. 145, 204501.

Senn, M. S., Keen, D. A., Lucas, T. C. A., Hriljac, J. A. \& Goodwin, A. L. (2016). Phys. Rev. Lett. 116, 207602.
Shephard, J. J., Klotz, S., Vickers, M. \& Salzmann, C. G. (2016). J. Chem. Phys. 144, 204502.

Shiell, T. B., McCulloch, D. G., Bradby, J. E., Haberl, B., Boehler, R. \& McKenzie, D. R. (2016). Sci. Rep. 6, 37232.

Shilling, J. E., Tolbert, M. A., Toon, O. B., Jensen, E. J., Murray, B. J. \& Bertram, A. K. (2006). Geophys. Res. Lett. 33, L17801.

Sławiński, W. A., Zacharaki, E., Fjellvåg, H. \& Sjåstad, A. O. (2018). Cryst. Growth Des. 18, 2316-2325.

Soper, A. K. (2009). Mol. Phys. 107, 1667-1684.

Treacy, M. M. J., Newsam, J. M. \& Deem, M. W. (1991). Proc. R. Soc. London Ser. A, 433, 499-520.

Tucker, M. G., Keen, D. A., Dove, M. T., Goodwin, A. L. \& Hui, Q. (2007). J. Phys. Condens. Matter, 19, 335218.

Turneaure, S. J., Sharma, S. M., Volz, T. J., Winey, J. M. \& Gupta, Y. M. (2017). Sci. Adv. 3, eaao3561.

Warner, J. H., Rümmeli, M. H., Gemming, T., Büchner, B. \& Briggs, G. A. D. (2009). Nano Lett. 9, 102-106.

Whale, T. F., Clark, S. J., Finney, J. L. \& Salzmann, C. G. (2013). J. Raman Spectrosc. 44, 290-298.

Willhammar, T. \& Zou, X. (2013). Z. Kristallogr. Cryst. Mater. 228, $11-27$.

Zimmermann, I. \& Johnsson, M. (2015). Solid State Sci. 40, 67-70. 